\title{
Review: antidepressant plus antipsychotic increases clinical response rates in psychotic depression
}

Wijkstra J, Lijmer J, Balk FJ, et al. Pharmacological treatment for unipolar psychotic depression: systematic review and meta-analysis. Br J Psychiatry 2006;188:410-15.

What are the relative efficacies of antidepressants, antipsychotics, or combinations of such drugs, for psychotic depression?

\section{METHODS}

Design: Systematic review and meta-analysis.

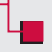

Data sources: Cochrane Central Register of Controlled Trials (search date not reported), MEDLINE (1966-2004), EMBASE (1980-2004), and trials known to the authors.

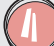

Study selection and analysis: Randomised controlled trials (RCTs) of antidepressant and/or antipsychotic treatment for people with psychotic depression (unipolar major depressive disorder with a current episode featuring psychotic symptoms) This included RCTs where not all participants had psychotic features, or where some participants had bipolar depression, but where results for the subgroup with unipolar psychotic depression were reported separately. RCTs with less than $20 \%$ of participants with bipolar depression were also included. Meta-analysis was carried out using RevMan 4.2 software.

Outcomes: Clinical response (as defined by individual studies based on improvement on observer-rated symptom measures, for example $\geqslant 50 \%$ reduction in symptom severity on Hamilton Rating Scale for Depression); withdrawal rate.

\section{MAIN RESULTS}

Ten RCTs met the inclusion criteria $(n=548)$. There was no significant difference between antidepressant (amitryptyline) monotherapy and placebo in clinical response (1 RCT, 27 people; RR 8.4, $95 \%$ CI 0.5 to 142.3 ), or between antipsychotic monotherapy and placebo (2 three-arm RCTs, 249 people; RR 1.1, 95\% CI 0.7 to 1.7 ). However, the combination of antidepressant (fluoxetine) plus antipsychotic (olanzapine) improved clinical response rates compared with placebo ( 1 three-armed RCT, 124 people, RR $1.9,95 \%$ CI 1.2 to 2.8 ). There was no significant difference in response between antidepressant (amitriptyline) monotherapy and antipsychotic (perphenazine) monotherapy (1 three-arm RCT, 58 people; RR 2.1, 95\% CI 0.6 to 6.8). A combination of an antidepressant plus an antipsychotic was more effective than antipsychotic alone (3 RCTs, 188 people; RR $1.9,95 \%$ CI 1.2 to 2.8 ), but was not more effective than antidepressant alone (2 RCTs, 77 people; RR 1.4, 95\% CI 0.9 to 2.4). There were no significant differences in withdrawal rates between any of the treatments.

\section{CONCLUSIONS}

The combination of antidepressant plus antipsychotic improves clinical response rates compared with placebo, and compared with antipsychotic alone in people with psychotic depression. Antidepressant plus antipsychotic was not significantly different For correspondence: Dr J Wijkstra, University Medical Centre Ütrechi HP 801.206, PO Box 85500, 3508 GA, Utrecht, the Netherlands; i.wijkstra@azu.nl

Sources of funding: Wyeth and AstraZeneca. from antidepressant alone, although the comparison of antidepressant monotherapy versus placebo did not show a statistical difference in clinical response rates.

\section{NOTES}

Some comparisons may have been underpowered to detect clinically relevant differences between treatments, as there were few RCTs and they had small sample sizes. The trials differed in their definitions of "clinical response", and were clinically heterogeneous.

\section{Commentary}

This systematic review compared the clinical effectiveness of different pharmacological strategies for people with psychotic depression. Although only a minority of patients with major depression experience psychotic symptoms, psychotic depression is a very important clinical entity because it is marked by greater severity, greater incapacity and longer duration of episodes than non-psychotic depression. 'This review identified only 10 randomised controlled trials (RCTs); more than 100 participants were enrolled in two trials only, and only three studies followed patients for a period of at least eight weeks. Additionally, the rate of withdrawals was considerably high (up to $50 \%$ in trials with longer follow-up), despite the fact that dosages of psychotropic drugs were clinically reasonable. This suggests that RCTs in this field are difficult to carry out and that psychotic depression remains an under-investigated topic. It is possible that the pharmaceutical industry is not interested in conducting trials in a condition that is not considered an independent diagnostic category, and therefore a specific indication for treatment may hardly be obtained by regulatory authorities. As the authors state in the text, more evidence should be collected urgently, but this field is commercially unattractive.

The main clinical finding is that there is no evidence that the combination of an antidepressant plus an antipsychotic is more effective than an antidepressant alone. This is in contrast with American Psychiatric Association and National Institute for Health and Clinical Excellence guidelines, where a combination strategy is recommended as first-line treatment in people with psychotic depression. ${ }^{2} 3$ However, antipsychotics are associated with troublesome adverse effects (such as extrapyramidal side effects, hyperprolactinaemia, seizures, hypotension, anticholinergic effects, weight gain, cardio-toxic effects, metabolic syndrome) and, consequently, an evidence-based approach to the management of these people should consider antidepressant monotherapy initially, and then a combination of antidepressant and antipsychotic if no response is achieved.

Andrea Cipriani, MD and Corrado Barbui, MD Department of Medicine and Public Health, Section of Psychiatry and Clinical Psychology, University of Verona, Verona, Italy

1 Coryell W. The treatment of psychotic depression. J Clin Psychiatry 1998;59(Suppl 1):22-9.

2 American Psychiatric Association. Practice guideline for the treatment of patients with major depressive disorder (revision). Washington, DC: American Psychiatric Association, 2000.

3 National Institute for Health and Clinical Excellence. Clinical Guideline 23. Depression. Management of depression in primary and secondary care. London: HMSO, 2004. 